Kasagi, J., et al., Energetic Protons and alpha Particles Emitted in 150-keV Deuteron Bombardment on Deuterated Ti. J. Phys. Soc. Japan, 1995. 64(3): p. 777-783.

\title{
Energetic Protons and a Particles Emitted in 150-keV Deuteron Bombardment on Deuterated Ti
}

\author{
Jirohta KASAGI, Tsutomu OHTSUKI, Keizo IsHII $^{1}$ and Masayuki HIRAGA ${ }^{2}$ \\ Laboratory of Nuclear Science, Tohoku University, \\ Mikamine, Taihaku-ku, Sendai 982 \\ ${ }^{1}$ Cyclotron and RI Center, Tohoku University, Aramaki, Aoba-ku, Sendai 980 \\ ${ }^{2}$ Department of Chemistry, Tohoku University, Aramaki, Aoba-ku, Sendai 980
}

(Received September 7, 1994)

\begin{abstract}
Energetic charged particles have been measured in the bombardment of $150-\mathrm{keV}$ deuterons on deuterated Ti. Protons and $\alpha$ particles were observed with energies up to $\sim 17$ and $\sim 6.5$ $\mathrm{MeV}$, respectively, which can never be attained in the $\mathrm{D}+\mathrm{D}$ reaction. A bump structure at around $14 \mathrm{MeV}$ seen in the proton spectrum can be well explained as emitted in the sequential reaction involving three deuterons. However, protons and $\alpha$ particles distributed continuously up to the maximum energies can never be understood as products of the conceivable nuclear reactions.
\end{abstract}

KEYWORDS: D+D reaction in Ti, high energy proton emission, $\alpha$-particle emission, 3D reactions, sequential reaction

\section{§1. Introduction}

In a series of experiments on bombardments of low energy deuterons on deuterated Ti for a study of the low energy $\mathrm{D}+\mathrm{D}$ reaction in metal, we have observed energetic charged particles which cannot be explained as emitted in usual nuclear reactions. They are protons with energies up to $\sim 17 \mathrm{MeV}$ and $\alpha$ particles with energies up to $\sim 6.5 \mathrm{MeV}$. In this paper, we point out how the observed energy spectra indicate that a reaction involving three deuterons occurs in the bombardment; a bump seen in the proton spectra can be well understood, whereas the mechanism of emitting the continuous protons and $\alpha$ particles is not completely clear at present time.

\section{§2. Experiment}

Targets were prepared as follows. $\mathrm{D}_{2}$ gas was absorbed by various Ti rods $(99.5 \% \mathrm{Ti} ; 10$, 8 , and $6 \mathrm{~mm}$ in diameter and $30 \mathrm{~mm}$ in length) and plates $(10 \mathrm{~mm} \times 30 \mathrm{~mm} \times 2 \mathrm{~mm})$, which were manufactured by Nippon Mining Co., Ltd. A cylindrical vessel $(16 \mathrm{~mm}$ in inner diameter and $10 \mathrm{~cm}$ in length) made of inconel metal was used for the gas absorption, and was set in an electric oven. Typical contaminants in Ti are listed in Table I. The vacuum line was made of stainless steel and was evacuated by a turbo molecular pump. Before admitting $\mathrm{D}_{2}$ gas, a Ti rod or plate was loaded into a vessel and degassed by heating the vessel at around $800^{\circ} \mathrm{C}$ in vacuum of $10^{-5} \mathrm{~Pa}$ for at least 20 hours. After that, the vessel was cooled down to about $600^{\circ} \mathrm{C}$ and then was filled with the $\mathrm{D}_{2}$ gas. The system was refilled with the gas again and again until the 
absorption was saturated. The average atomic ratio $\mathrm{D} / \mathrm{Ti}$ was obtained by weighing the rod before and after the $\mathrm{D}_{2}$ gas loading. The rods and plates with the atomic ratio larger than 1.0 were used as targets.

Table I. Contaminants in Ti metal.

\begin{tabular}{cl}
\hline Element & Quantity (\%) \\
\cline { 1 - 2 } $\mathrm{C}$ & 0.03 \\
$\mathrm{~N}$ & 0.015 \\
$\mathrm{O}$ & 0.2 \\
$\mathrm{Al}$ & 0.03 \\
$\mathrm{Si}$ & 0.03 \\
$\mathrm{Ca}$ & 0.002 \\
$\mathrm{Cr}$ & 0.005 \\
$\mathrm{Mn}$ & 0.01 \\
$\mathrm{Fe}$ & 0.15 \\
$\mathrm{Cu}$ & 0.0005 \\
$\mathrm{Sn}$ & 0.02 \\
\hline
\end{tabular}

The target was bombarded with a deuteron beam obtained from the Cockcroft-Walton accelerator at the Department of Chemistry at Tohoku University. Deuterons were ionized in an RF-type ion source and accelerated up to $150 \mathrm{keV}$. After the acceleration, the beam was bent by 15 degrees by a dipole magnet in order to select the $\mathrm{D}^{+}$beam. The beam passed through a collimator of $3 \mathrm{~mm}$ diameter set at the entrance of a scattering chamber. The target was set at the center of a chamber and was irradiated. Since the beam was stopped in the target, the electric current from the target was monitored and integrated during the run. Typical beam intensity was about $2 \mu \mathrm{A}$.

Charged particle measurements were carried out with different detector setups. In the early stage, we mainly focused on energetic protons in the bombardment. For this purpose, either a surface barrier Si detector ( $2 \mathrm{~mm}$ in thickness and $25 \mathrm{~mm}^{2}$ in area) or a Li-drifted Si detector (5 $\mathrm{mm} \times 100 \mathrm{~mm}^{2}$ ) was used with an $\mathrm{Al}$ absorber; high energy protons were identified by changing the thickness of the absorber. A $\Delta E-E$ counter telescope consisting of $20-\mu \mathrm{m}$ and 1-mm thick Si detectors was used later in order to measure $\alpha$ particles with energies of several $\mathrm{MeV}$. In this case, $2-\mu \mathrm{m}$ thick $\mathrm{Al}$ foil was placed in front of the $\Delta E$ detector to prevent $\delta$-rays and scattered deuterons from hitting the detector. The detector was placed at a distance between 1.5 and $3 \mathrm{~cm}$ from the target and at several angles between $90^{\circ}$ and $155^{\circ}$ with respect to the beam direction. The counting rate of the detector was typically about $100 \sim 200$ counts/sec. Energy resolution of the detectors was checked with an ${ }^{241} \mathrm{Am}$ source and was about $30 \mathrm{keV}$ for $5.48 \mathrm{MeV} \alpha$ particles, which was also served for the energy calibration. 


\section{§3. Observation of Protons and $\alpha$ Particles}

Very energetic particles were observed in the bombardment. In Fig. 1(a) is shown such a spectrum measured at $135^{\circ}$ with a $15-\mu \mathrm{m}$ thick $\mathrm{Al}$ absorber. A huge peak appearing at about 2.45 $\mathrm{MeV}$ is attributed to protons emitted in the $D(d, p) T$ reaction. Because of the steep fall of the reaction cross section for lower incident energies, the reaction mainly occurs in thin layers near the surface so that the observed peak is sharp; a simple kinematical consideration gives a broader peak between 2.74 and $3.02 \mathrm{MeV}$ whereas the actual energy of the peak is $2.75 \mathrm{MeV}$ after correction for energy loss in the $\mathrm{Al}$ absorber. Events due to double and triple pileups of the protons are distributed up to about 4.9 and $7.4 \mathrm{MeV}$, respectively, where sharp edges are clearly seen in the spectrum. In addition to these normal events, events up to $17 \mathrm{MeV}$ are also seen. They are neither pileups nor events produced by the protons in the detector as proved by Fig. 1(b) which shows the spectrum measured with a $200-\mu \mathrm{m}$ thick $\mathrm{Al}$ foil stopping the $2.75-\mathrm{MeV}$ protons. As seen, the huge proton peak and the pileups disappear, and events due to electronic noises are inevitably seen at the low energy region $(E<3 \mathrm{MeV})$, although its counting rate is quite small $(<0.1$ counts/sec). However, at the higher energy region, the similar structure as observed in Fig. 1(a) remains with a energy shift to lower energies. The amount of its reduced energy clearly indicates that these high energy particles are protons emitted in the target.

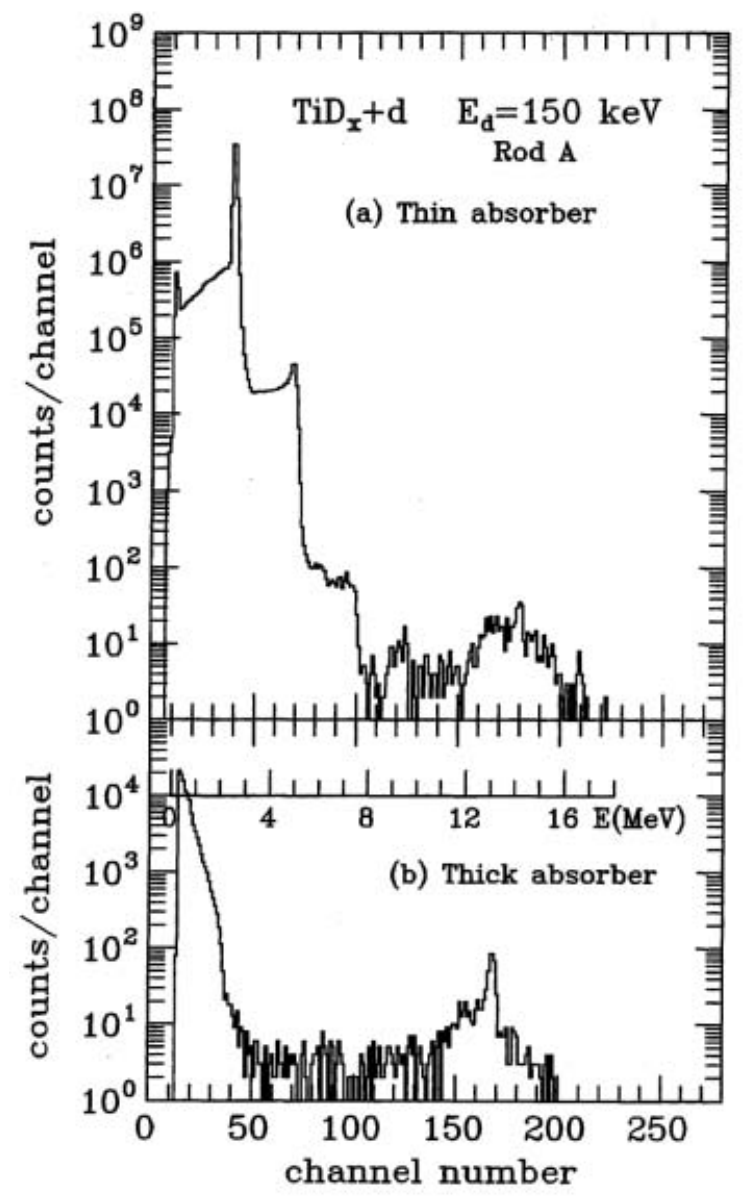

Fig. 1. Charged particle spectra obtained in the $150-\mathrm{keV}$ deuteron bombardment on TiD, at $135^{\circ}$; (a) measured with a 15-fim thick $\mathrm{Al}$ absorber and (b) with a 200-,um thick $\mathrm{Al}$ absorber. The energy scale represents energy of charged particles after passing through the absorber. 
The characteristics of the high energy proton structure are these: a broad bump ranging from 12.5 to $16.5 \mathrm{MeV}$, a sharp peak at $14.1 \mathrm{MeV}$, and a continuum up to $\sim 17 \mathrm{MeV}$ where the bump and the peak are superimposed. The sharp peak was interpreted as protons emitted in the ${ }^{3} \mathrm{He}(d, p){ }^{4} \mathrm{He}(Q=18.35 \mathrm{MeV})$ reaction, ${ }^{1)}$ since the peak energy just follows the kinematical prediction at detected angles of $90^{\circ}, 110^{\circ}$ and $135^{\circ}$; the interpretation requires an anomalous concentration of ${ }^{3} \mathrm{He}$ in the target before the bombardment began as discussed in ref. 1 . However, we do not discuss the peak in this paper, since a systematic study of the peak is very difficult because of its low probability of occurrence; we have had only four cases which show the peak clearly, out of more than 100 bombardments on various $\mathrm{TiD}_{\mathrm{x}}$. By contrast, the bump and the continuum which will be discussed, later, always appear in any measurements on $\mathrm{TiD}_{\mathrm{x}}$ as long as $x>$ l.2. In Fig. 2 , high energy part of the proton spectra $\left(E_{\mathrm{p}}>6 \mathrm{MeV}\right)$ measured at $110^{\circ}, 135^{\circ}$ and $155^{\circ}$ are shown. Since the spectra in Fig. 2 were obtained with different targets from the one in Fig. 1, they do not show the sharp peak at $14.1 \mathrm{MeV}$, but do show the bump and continuum; its line shape and the maximum energy depends on the detection angle. In Figs. 2(b) and 2(c), yields of protons below $7.5 \mathrm{MeV}$ suddenly increase because of the pileup protons, whereas the spectrum in Fig. 2 (a) does not show such increase since it was measured with a thick Al absorber.

Figure 3 shows a scatter plot of $\Delta E$ vs. $E$ measured at $135^{\circ}$ in the bombardment on $\mathrm{TiD}_{\mathrm{x}}$ Tritons and ${ }^{3} \mathrm{He}$ particles emitted in the $\mathrm{D}+\mathrm{D}$ reaction cannot punch through the $\Delta E$ detector. Protons from the $\mathrm{D}(d, p)$ T reaction as well as their pileups are seen as a heavy locus in the lowest part of the figure, although the low energy part of $\Delta E$ is cut, where a huge peak is expected at about $(E, \Delta E)=(850,50)$ channels. A peak at about $(850,250)$ channels is due to a proton accidentally accompanied by a triton which stops in the $\Delta E$ detector. No loci corresponding to other $Z=1$ particles, deuterons and tritons, were not identified in the region just above the proton locus where observed events are considered to be due to the large tail of protons. Observed is a broad locus consisting of several tens of events at larger $\Delta E$, and which was actually assigned to be $\alpha$ particles based on the observed energy loss. The assignment was also confirmed by the fact that $\alpha$ particles from an ${ }^{241} \mathrm{Am}$ source make a spot on the locus. No ${ }^{3} \mathrm{He}$ particles were observed, whose locus is expected, if exists, to have about $25 \%$ lower $\Delta E$ than that of $\alpha$ particles. 


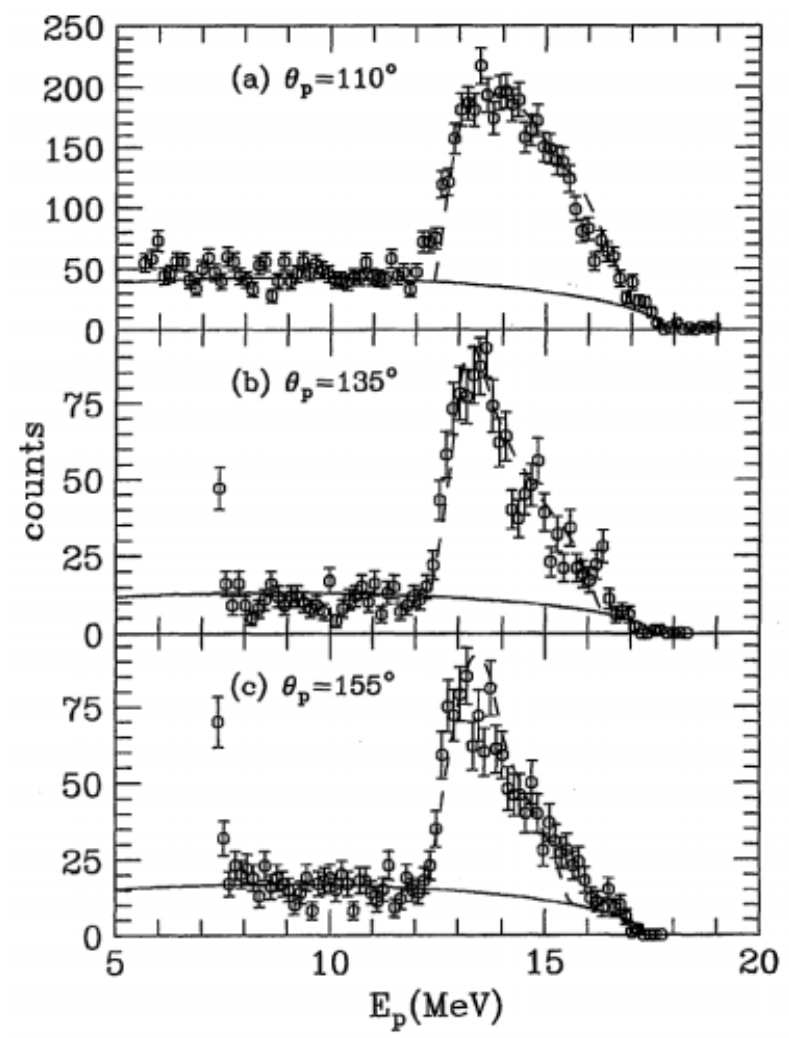

Fig. 2. High energy proton spectra measured at (a) $110^{\circ}$, (b) $135^{\circ}$ and (c) $155^{\circ}$. Solid lines represent available phase spaces of protons emitted in the three-body reaction. Dashed lines are the calculated spectral shapes of protons emitted in the sequential reaction and are superimposed on the solid lines. The spectrum shown in (a) was measured with a $200-\mu \mathrm{m}$ thick $\mathrm{Al}$ absorber, whereas the spectra in (b) and (c) were measured with a 15$\mu \mathrm{m}$ one.

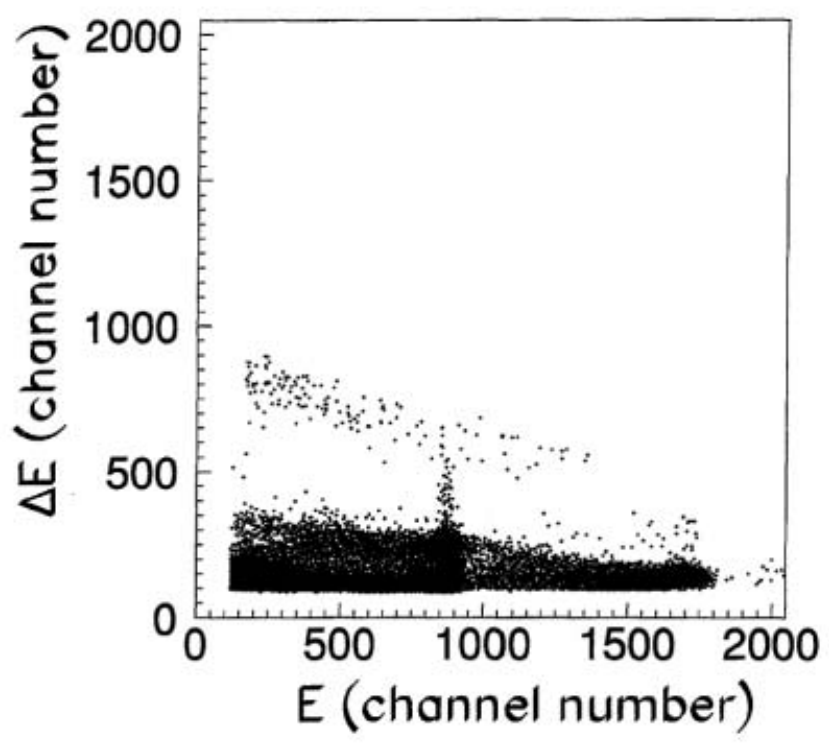

Fig. 3. Scatter plot of $E$ versus $\Delta E$, measured in the $150-k e V$ deuteron bombardment on $\mathrm{TiD}_{\mathrm{x}}$ at $135^{\circ}$. $\mathrm{A}$ broad locus at higher $\Delta E$ region is due to $\alpha$ particles. 
Energy spectra of the $\alpha$ particles at $135^{\circ}$ and $155^{\circ}$ were deduced by setting a banana gate on the two-dimensional spectra. These spectra are shown with open circles in Fig. 4. As shown, $\alpha$ particles are observed up to $\sim 6.5 \mathrm{MeV}$ (those with energy lower than $4.5 \mathrm{MeV}$ were stopped in the $\Delta E$ detector). Figure 5 shows the ratio of the yield of $\alpha$ particles above $4.5 \mathrm{MeV}$ to that of the protons from the $\mathrm{D}(d, p)$ T reaction against the incident deuteron energy. Although the production rate is quite small, the ratio slightly increases as the increase of the incident energy.

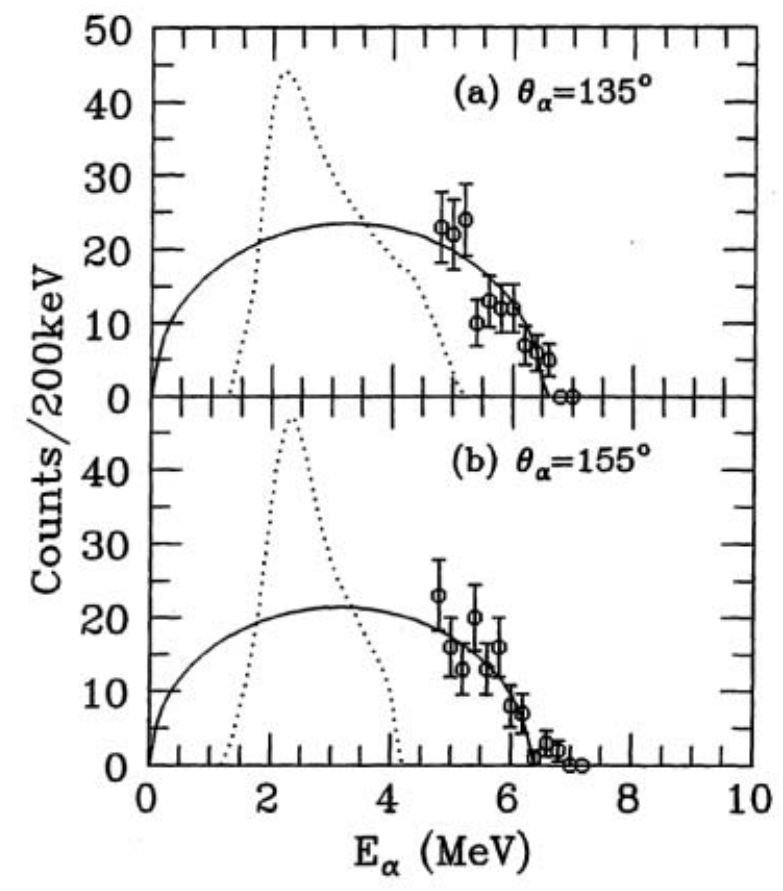

Fig. 4. Alpha-particle spectra measured at (a) $135^{\circ}$ and (b) $155^{\circ}$. Solid lines represent available phase spaces of $\alpha$ particles emitted in the three-body reaction. Dotted lines are the calculated spectral shapes of $\alpha$ particles emitted in the sequential reaction.

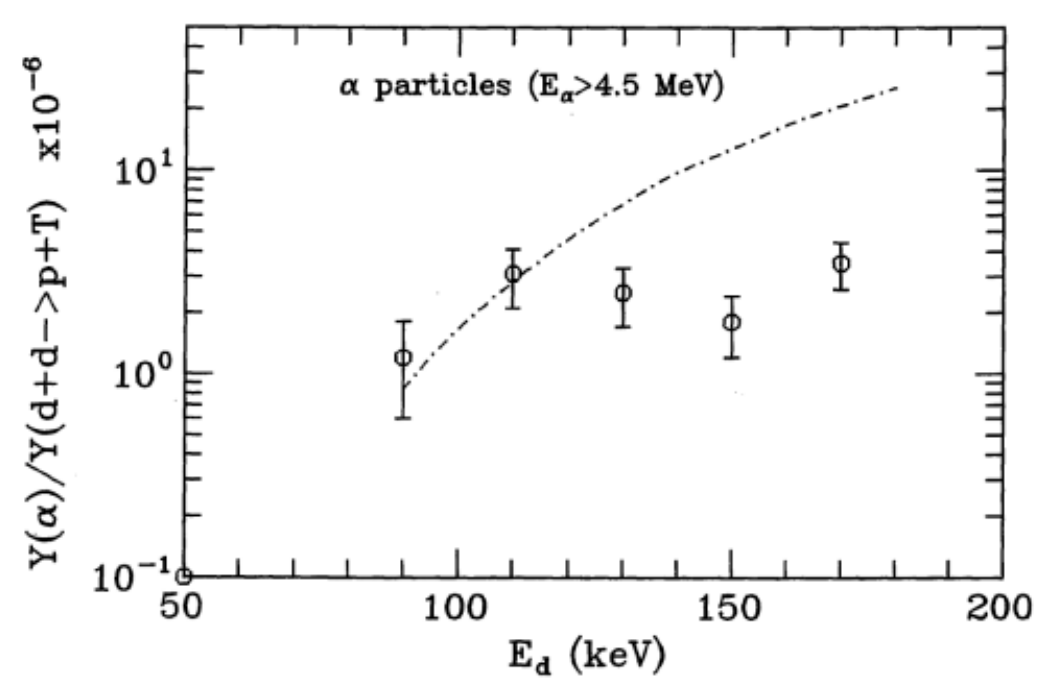

Fig. 5. Ratio of the yield of $\alpha$ particles to that of the protons emitted in the $D(d, p)$ T reaction against the incident energy. A dashed line shows the ratio of the Coulomb penetration factor for the $\mathrm{Li}+d$ reaction to that for the $D+D$ reaction (normalized to the data at around $90 \mathrm{keV}$ ). 
In order to check the possibility that the $\alpha$ particles are emitted in the secondary reactions induced by any materials in the target with the products of the primary $\mathrm{D}+\mathrm{D}$ reactions, we have irradiated the target directly with neutrons, protons and ${ }^{3} \mathrm{He}$ particles, and measured the charged particles. For neutron radiation, we used the same setup as the $\alpha$ particle measurement. Another $2-\mathrm{mm}$ thick $\mathrm{TiD}_{\mathrm{x}}$ plate was placed between the primary target and the detector. In this way, neutrons from the $\mathrm{D}+\mathrm{D}$ reaction can irradiate the other target. No $\alpha$ particles were observed for the irradiation with neutron dose of 10 times more than the experimental condition. $\mathrm{The}^{\mathrm{TiD}} \mathrm{x}$ target were directly bombarded with $3.3 \mathrm{MeV}$ proton and $1.5 \mathrm{MeV}^{3} \mathrm{He}$ beam obtained from a Van de Graaff accelerator at Tokyo Institute of Technology. The irradiated dose was more than 100 times of that of the products of the $\mathrm{D}+\mathrm{D}$ reaction. Again, no $\alpha$ particles were observed. Thus, the $\alpha$-particle production rate due to the secondary reactions induced by neutrons, protons and ${ }^{3} \mathrm{He}$ particles is less than $10^{-7}$ of the reaction rate of the $\mathrm{D}+\mathrm{D}$ reaction, i.e., at least one order of magnitude smaller than observed.

It should be noted, here, that neither protons nor $\alpha$ particles were observed in measurements without deuteron beams. Thus, high energy protons and $\alpha$ particles observed in the deuteron bombardments are not due to the cosmic-rays and $\alpha$-decays from $\mathrm{U}$ or Th existing in materials.

\section{§4. Discussion}

Reactions of the incident deuterons with Ti and deuterons cannot produce such high energy protons as observed up to $18 \mathrm{MeV}$. Moreover, those with the known contaminants in $\mathrm{Ti}$ metal which are listed in Table I cannot produce the high energy protons, either, since reaction $Q$-values are much smaller than $18 \mathrm{MeV}$. As shown in Fig. 1, the major reactions in the deuteron bombardment are the $\mathrm{D}+\mathrm{D} \rightarrow p+t(Q=4.03 \mathrm{MeV})$ and the $\mathrm{D}+\mathrm{D} \rightarrow n+{ }^{3} \mathrm{He}(Q=3.27 \mathrm{MeV})$ reactions. Although the secondary reactions of these reaction products have been naturally considered, no reactions can produce such high energy protons except for the $\mathrm{D}+{ }^{3} \mathrm{He}$ reaction.

For the broad bump observed between 12 and $16 \mathrm{MeV}$, protons are interpreted to be emitted in the $\mathrm{D}\left({ }^{3} \mathrm{He}, p\right)^{4} \mathrm{He}$ reaction $(Q=18.35 \mathrm{MeV})$ which sequentially occurs following the primary $\mathrm{D}\left(d,{ }^{3} \mathrm{He}\right) n$ reaction. In this case the ${ }^{3} \mathrm{He}$ particle ejected in the primary reaction reacts with deuterons at rest in the target. However, the protons emitted in the secondary reaction cannot form a sharp peak, because of the spread of energy and direction of the ${ }^{3} \mathrm{He}$. In order to verify this situation quantitatively, the spectral shape of the protons has been calculated for the sequential reaction. An excitation function of the cross section and angular distributions of the primary $\mathrm{D}\left(d,{ }^{3} \mathrm{He}\right) n$ reaction for $E_{\mathrm{d}}<150 \mathrm{keV}$ were taken from ref. 2 . Angular distributions of the secondary $\mathrm{D}\left({ }^{3} \mathrm{He}, \mathrm{p}\right)^{4} \mathrm{He}$ reaction were assumed to be isotropic in the CM frame for $E_{3-\mathrm{He}}<$ $1.33 \mathrm{MeV}$ (the maximum energy for the secondary reaction), and cross sections were estimated from the differential cross sections ${ }^{3)}$ and S-factors. ${ }^{2)}$ Values of deuteron and ${ }^{3} \mathrm{He}$ energy loss in Ti were taken from ref. 4. In Fig. 2, spectra measured at $110^{\circ}, 135^{\circ}$ and $155^{\circ}$ are compared with the calculations. Dashed lines in the figure are the calculated spectral shapes superimposed on the assumed continuum (discussed later) drawn with solid lines. As seen, the calculation reproduces the experimental shapes very well for each angle. Since the production rate of ${ }^{3} \mathrm{He}$ is proportional to the density of deuterons which are also the targets of the secondary reaction, the reaction rate of the sequential reaction is proportional to the square of the deuteron density in Ti. In order to reproduce the ratio of the yield of the bump to that of the peak of the $\mathrm{D}(d, p)$ T reaction 
(order of $10^{-5}$ ), the required $\mathrm{D} / \mathrm{Ti}$ ratio at the beam spot is more than 1.2. This explains why the bump was not easily seen for the targets with small $\mathrm{D} / \mathrm{Ti}$ ratio $(<0.6)$.

The fact that protons are observed with energies up to $\sim 17 \mathrm{MeV}$, as clearly seen in Fig. 2, is really anomalous. As already mentioned, none of the secondary reactions with neutron, triton or ${ }^{3} \mathrm{He}$ can produce such high energy protons. The yield of the continuum part shown with solid lines in Fig. 2 is about $10^{-6}$ of that of the protons from the $\mathrm{D}(d, p) t$ reaction. Protons emitted in the sequential $\mathrm{D}\left({ }^{3} \mathrm{He}, p\right){ }^{4} \mathrm{He}$ reaction are scattered in the tar-get and can contribute to the continuum in the energy region below the bump at $14 \mathrm{MeV}$. The yield of such events was estimated and was found to be one order of magnitude smaller than the observed one. The protons observed in the energy region above the bump can never be attributed to the scattered protons. Therefore, the question is the origin of the continuous protons existing up to $\sim 17 \mathrm{MeV}$.

Alpha-particle emission in the bombardment is quite anomalous, as well. As shown in Fig. 5, the yield of the $\alpha$ particles is quite small, again, about $10^{-6}$ of that of the protons produced in the $\mathrm{D}(d, p) T$ reaction. Although the reactions ${ }^{45,47} \mathrm{Ti}+d$ are kinematically possible to produce $\alpha$ particles up to $6.3 \mathrm{MeV}$ at $130^{\circ}$, the interpretation that the $\alpha$ particles are produced in these reactions can never explain the production rate and its incident energy dependence shown in Fig. 5. A simple consideration on the Coulomb barrier for the $\mathrm{Ti}+d$ reactions requires that the reaction rate should be at least 30 orders of magnitude smaller than the observed one and the incident energy dependence should be much steeper than in Fig. 5. The same argument rejects any possibility that the $\alpha$ particles are emitted in the reactions induced by the incident deuterons with contaminants in $\mathrm{Ti}$ which are listed in Table I. The predicted incident energy dependence of the production rate cannot be explained, even for the $Z=3$ target as shown with a dashed line in Fig. 5. It shows the calculated ratio of the reaction yield for the $\mathrm{Li}+d$ reaction to that of the $\mathrm{D}+\mathrm{D}$ reaction, normalized at $90 \mathrm{keV}$ of the incident energy.

As mentioned in $\S 3$, we have checked the possibility of the secondary reactions, experimentally, except for the triton induced reactions. The production rate is proportional to the number of the target nuclei as well as the reaction cross section. The largest case is the ${ }^{27} \mathrm{Al}(t$, $\alpha)^{26} \mathrm{Al}$ reaction which occurs in the $2-\mu \mathrm{m}$ thick $\mathrm{Al}$ absorber placed in front of the detector. We estimated the yield of $\alpha$ particles for the largest case. The total reaction cross sections were calculated by using an optical potential code ${ }^{5)}$ with several sets of potential parameter. ${ }^{6)}$ The calculated cross section for $0.7 \mathrm{MeV}$ triton which corresponds to the measurement at $135^{\circ}$ is only $0.01 \mathrm{mb}$. The expected total yield is 4 orders of magnitude smaller than the observed one. The spectral shape of emitted $\alpha$ particles in the sequential reactions in which the $\mathrm{D}\left({ }^{3} \mathrm{He}, \alpha\right) p$ and the $\mathrm{D}(d, t) p$ reaction follows the $\mathrm{D}\left(d,{ }^{3} \mathrm{He}\right) n$ and the $\mathrm{D}(\mathrm{rf}, t) p$ reaction was calculated using the same program used to calculate the proton spectrum. The result also gave a bump in the $\alpha$ particle spectrum. In this case, however, the expected bump lies in a lower energy region than observed, as shown with dotted lines in Fig. 4. Thus, the observed $\alpha$ particles are not explained as the products of the sequential reactions involving another deuteron in the target.

Since $\alpha$ particles can be produced in the primary reactions with the contaminants or in the sequential reactions mentioned above, they can exist in the target and can be knocked out by the $14-\mathrm{MeV}$ protons and neutrons. However, the maximum energy of the $\alpha$ particles in such cases is only $2.8 \mathrm{MeV}$. Thus, the knock-out reaction cannot explain the observed $\alpha$ particles.

We have inferred that the anomalous energetic protons and $\alpha$ particles are attributed to a common origin. Of particular interest is the fact that the observed maximum energies up to 
which the anomalous particles are emitted are always larger than possible from the sequential reaction. Thus, we have speculated that the protons and $\alpha$ particles are emitted in the reaction where the bombarded deuteron reacts with two deuterons in the target, without forming a real intermediate state. The incident deuteron first interacts with a deuteron to form a virtual intermediate state, which subsequently interacts with another deuteron and produces neutron, proton and $\alpha$ particle. In this case, the available energy is shared with the three particles in the final state; the maxi-mum energy of each particle can be larger than that of the sequential reaction.

Based on this very naive picture, we have calculated the phase spaces of protons and $\alpha$ particles being available in the $\mathrm{D}+\mathrm{D}+\mathrm{D} \rightarrow p+n+\alpha$ reaction $(Q=21.62 \mathrm{MeV})$. In the calculation, we assumed that the $150-\mathrm{keV}$ deuteron reacts with two deuterons whose center of mass is at rest. The results of the calculation are drawn with solid lines in Fig. 2 and Fig. 4. Since the calculation depends only on the kinematical factor, yields are normalized with the data. As seen, the calculation explains both spectra very well; i.e., the maximum energies and their spectral shapes. Thus, the observed spectra indicate the possibility that the incident deuteron interacts with two other deuterons to produce $n, p$ and $\alpha$ without forming any real nuclear state in an intermediate stage.

The integrated yields of the anomalous protons and $\alpha$ particles are about $10^{-5}$ times of that of the protons of the $\mathrm{D}(d, p)$ T reaction. The reaction rate of the hypothetical three-body reaction can be factorized as $R_{d d d}=A \times P\left(r_{0}\right)$, where $P\left(r_{0}\right)$ is the probability that the two deuterons in the target are within a distance of $r_{0}$. If the reaction proceeds only through the nuclear interaction, then $r_{0}$ should be order of a nuclear radius, about $10 \mathrm{fm}$. In order to obtain a rough value of $R_{d d d}$, we assume that $A$ is equal to the reaction rate of the usual $\mathrm{D}+\mathrm{D}$ reaction; the bombarded deuteron interacts with one of the deuterons of the pair and the virtual intermediate state immediately interacts with the other deuteron, resulting in neutron, proton and $\alpha$ particle in the final state. The probability to find the two deuterons at $r_{0}$ is basically determined by the Coulomb penetration factor. Ichimaru et al. calculated the probability in $\mathrm{Pd}$ and $\mathrm{Ti}$, including an enhancement due to the many-particle processes of the screened deuterons. ${ }^{8)}$ The ratio of the three-body reaction rate to the two-body one is then estimated to be order of $\sim 10^{-17}$, which is far below the experimental ratio of $10^{-5}$. Thus, various factors which is not included in the model calculation should be considered. For example, high concentration of deuteron realized by the continuous bombardment on highly deuterated metal would cause a flow of deuterons; the situation cannot be a static one. In a very low energy region, the penetration factor of deuterons might be much less than the expected one from the adiabatic approximation as shown for fusion reactions with light nuclei. ${ }^{9)}$ A possibility that the second step interaction is the electromagnetic one increases the value of $r_{0}$ and hence $P\left(r_{0}\right)$ can be much larger.

\section{§5. Conclusion}

In summary, in the $150-\mathrm{keV}$ deuteron bombardment on highly deuterated $\mathrm{Ti}$, we have observed energetic protons and $\alpha$ particles which cannot be emitted in the $\mathrm{D}+\mathrm{D}$ reaction. A bump structure at around $14 \mathrm{MeV}$ seen in the proton spectrum can be well explained as emitted in the sequential reaction involving three deuterons. However, the continua of protons and $\alpha$ particles up to $\sim 17$ and $\sim 6.5 \mathrm{MeV}$, respectively, cannot be explained as the products of the conceivable two-body nuclear reactions. The possibility of attributing the protons and the $\alpha$ particles to the three-body reaction, $\mathrm{D}+\mathrm{D}+\mathrm{D} \rightarrow p+n+\alpha$, is suggested, since it can explain the maximum energies 
and spectral shapes. However, this possibility requires an anomalously large enhancement factor which is not understood at present. Therefore, further experimental studies, especially coincidence measurements of protons and $\alpha$ particles, are highly desirable.

\section{Acknowledgements}

The author would like to thank to Professor Yoshihara for facilitating the use of the accelerator. This work was partially supported by a Grant-in-Aid for Co-operated Research (A) No. 02305015 from the Ministry of Education, Science and Culture.

\section{References}

1. J. Kasagi, K. Ishii, M. Hiraga and K. Yoshihara: Proc. $3^{\text {rd }}$ Int. Conf. Cold Fusion, Nagoya, 1992, ed. H. Ikegami (Universal Academy Press, Tokyo, 1993) p. 209.

2. A. Krauss, H. W. Becker, H. P. Trautvetter, C. Rolfs and K. Brand: Nucl. Phys. A 465 (1987) 150.

3. J. L. Yarenell, R. H. Lovberg and W. R. Stratton: Phys. Rev. 90 (1953) 292.

4. J. F. Ziegler: The Stopping and Ranges of Ions in Matter (Pergamon Press, 1980) Vol. 3 and 4.

5. Optical Potential Code ALPS.

6. C. M. Percy and F. G. Perey: At. Data Nucl. Data Tables 17 (1976) 1.

7. S. Ichimaru: Rev. Mod. Phys. 65 (1993) 255, and references therein.

8. S. Englster, G. Raimann, C. Angulo, U. Greife, C. Rolfs, U. Schroder, E. Somorjai, B. Kirch and K. Langanke: Phys. Lett. B 279 (1992) 20. 